Accepted version of Scheeres, H., Solomon, N., Boud, D., and Rooney, D. (2010). When is it OK to learn at work? The learning work of organisational practices, Journal of Workplace Learning, 22, 1\&2, 13-26.

\title{
When is it OK to learn at work? The learning work of organisational practices
}

\author{
Hermine Scheeres, Nicky Solomon, David Boud and Donna Rooney \\ Research Centre for Learning and Change \\ University of Technology, Sydney
}

\begin{abstract}
Purpose

While the term 'learning' is ubiquitous in organisations, it is used and understood in very different ways. This paper examines the use of 'learning' through what we have termed 'integrated development practices'. These are common organisational practices that both enhance organisational effectiveness and contribute to organisational and employee learning.

Design/methodology

This paper analyses the ways in which learning and being a learner were talked about and enacted with regard to one of the integrated development practices identified in a study of four different organisations - safety practices, and how learning and being a learner regarding safety were legitimate in one of the organisations. Data is drawn from semi-structured interviews with members of a variety of workgroups in one major division of the organisation.
\end{abstract}

\section{Findings}

Interviewees' responses reflected that learning was fully embedded as an accepted part of a necessary function of the organisation. This use of a learning discourse is discussed in the light of findings from an earlier study on informal learning at work that suggested that learning and the identity of being a learner were sometimes resisted in the everyday culture of work.

Originality/value

Using the theorisations of practice of Schatzki $(2005 ; 2006)$ and the lifelong education framework of Delors (1996) the paper discusses the implications of these findings to examine when it is acceptable to articulate learning as part of work and be identified as a learner at work.

Keywords: workplace learning; organisational learning; workplace learning discourses; workplace practices; practice theory

\section{Introduction}

Learning discourses pervade contemporary workplace and organisational literatures. This foregrounding of learning has shifted, or at least disturbed, more traditional discourses of education and training in the context of work. As such, the shift has opened up exploration of work/learning and worker/learner links. Thus, we know that 
learning occurs 'at' work in many different ways, and that all workers are involved in learning. In this paper, our underlying question is: when is it okay to learn and be a learner at work? We address this question through an investigation of how discourses of learning and being a learner - both explicit and implicit - are accepted and integrated into how employees represent themselves and their work.

This question is part of our ongoing exploration of what learning work organisations do. That is, how is learning deployed within organisations and how is learning regarded and talked about. In particular, we are concerned with learning that takes place through organisational practices when there is no teacher, trainer or explicit learning agenda. This is a central focus of our Australian Research Council funded study Beyond training: integrated development practices in organisations.

Our research is located in-between work-related education and training courses and everyday learning at work in that we are concerned with organisationally-sanctioned and named practices that are initiated explicitly to enhance organisational effectiveness. We have called these organisational practices 'integrated development practices' (IDPs) which we define as ones that: (1) facilitate learning in a way that is embedded in work; (2) are independent of formal training programs and are not typically described in terms of training or education; and, (3) are managed or implemented by people whose primary job function is not training or learning. The IDPs are 'integrated' in that they constitute part of the 'normal' work of an organisation, yet they are also distinctive practices that are named and described in an organisation's written materials and/or part of employees' experiences of what goes on in their organisation. We use 'development' to refer particularly to the overall aim of the practices to enhance the success of the organisation. Finally, following Schatzki (2005; 2006), we see 'practices' as work-related doings and sayings made up of both structure and action, and organised around shared understandings. In a later section of this paper, we elaborate on how we are working with contemporary notions of practice as a conceptual frame to further understand how learning is deployed through work. In the four research sites that form our study, we have identified a range of IDPs including performance appraisal, employee induction, acting up/acting across (positions), projects and team meetings.

While our broad focus is on IDPs, here we take up the question of whether learning and being a learner are accepted at work, and use this to illuminate the ways in which IDPs incorporate learning. The paper begins by locating our study and this paper within the wider field of work and learning. It focuses then on conceptual and framing ideas from Schatzki and Delors that we have used, and how we framed our findings for collaborative discussions with the research site organisation. As we wished to involve the organisation actively in our research we sought ways in which learning in the organisation could be communicated effectively to those who had invited us in. Drawing out and making explicit how learning is related to practice theory is part of our ongoing research work, however, to ensure fruitful discussions and feedback we identified Delors' (1996) notion of the four pillars of education as a useful way of framing our findings regarding learning for workers and managers.

The main part of the paper discusses how an IDP, safety practices, in one organisation - a large utility company in NSW, Australia - is deployed, and it draws out how learning is talked about and legitimised as part of the IDP. The company, that 
we have named SupplyCo, is introduced, and the centrality of (changing) safety practices to its operation and identity is discussed. The data presented is taken from interviews with managers and workers within the utility, and analysed in terms of the discourses they used with respect to the range of safety practices in the organisation. In particular, we explore how employees embed, either explicitly or implicitly, notions and experiences of learning safety practices in their interview responses. We conclude by returning to our question about how learning is regarded within the organisation, and how we presented workplace learning in our discussions with the organisation.

\section{Locating the study: work and learning research}

In this paper we locate learning as embedded in the practices of work. As researchers, our study of learning and work usually comes from a tradition of research on learning and learners. Within this tradition the research focus is either on work-related learning where learning occurs through structured courses on and off-site, or on everyday learning at work where learning is considered to be involved in the normal conduct of work. However, in this study, while influenced by theorisations within that tradition, we are moving outside pedagogical discourses or interventions (Bereiter 1994:21, Saugstad 2005, Boud 2006), to engage with learning at work integrated with collections of practices that constitute particular kinds of everyday work.

Our interest in learning at work is prompted by employees who, when asked to nominate where they learn most, overwhelming point to their workplace as a major site for their learning. Similarly, employers, when asked how their employees learn, suggest that learning through 'daily practice means a great deal to the employee's ability to do a good job' (Skule and Reichborn 2002:14-15). Theorists have taken up this idea by trying to understand how and to what extent workplaces are and can be made learning places (Ellström 2001). Indeed there is now an extensive literature on this topic with educationalists, sociologists, anthropologists, psychologists, and management and economic theorists bringing their viewpoints and conceptual insights to bear on the domain of learning at work. New concepts have entered the arena of workplace learning including 'learning organisations' (Senge 1990), 'situated learning' and 'communities of practice' (Lave and Wenger 1991) 'expansive learning' (Engeström 2001), 'curriculum connectivity' (Guile and Griffiths 2001), 'workplace pedagogy' (Fuller and Unwin 2002) 'learning-conducive work' (Skule and Reichborn 2002), 'everyday learning' (Boud and Solomon 2003), and 'learningnetworks' (Poell, Chivers and Van der Krogt 2000).

While the diversity of these new terms reveals disparate theoretical positions, as Hodkinson (2004:12) points out, there is considerable agreement in the literature that 'learning at work cannot be separated out from the everyday working practices of the workplace'. We understand learning for work as something that can be seen as separate from yet related to work. We also acknowledge that there is a sense in which learning at or in work is part of what constitutes work practices. In other words, work practices are not simply a series of repetitions of previous practices (though there may be some repetition of particular activities, as well as routines or protocols). Rather, work practices are more than activities in that they involve complex bundles of doings and sayings that change over time (Schatzki 2006), and these changes involve learning. We maintain it is useful to tease out how learning is implicated in these 
bundles of doings and sayings, and we are interested in specific kinds of doings and sayings - IDPs. Our focus on IDPs as recognisable and often documented practices that explicitly aim to improve work and worker productivity seeks to contribute a new dimension to the theorisations and investigations of organisational practices and learning. Our interest is identifying and explaining the learning that occurs within IDPs in order to reveal relationships between people, between people and systems, processes and material arrangements.

In adopting this position we acknowledge the useful contributions of writers across a range of fields such as those mentioned above. Our concern with practices however does not specifically draw on these writers as we seek to gain different understandings of the relationships that exist between organisations, work, workers and learning. Conventionally, learning and work are discursively constructed in distinctive ways. Learning has most often been associated with the world of education and training. It has focused primarily on knowledge and skills as 'things' that are able to be learned independent of a specific context. Further, typically learning has been judged or assessed in terms of the individual. Working is primarily located and judged in terms of effects on an organisation and its goals. We are examining practices that straddle these domains in order to further understand how working and learning are integrated generally, but mainly to draw out how practices designated as organisationally important - IDPs - can be understood as learning practices. In addition, we have found that change is a significant element associated with IDPs. As Saugstad (2005) writes 'Learning and activities in practice are closely connected, since learning is understood as changing positions in a changing social practice'. Learning can therefore be understood as being 'located within the context of activity and/or in a social practice' (p.351).

\section{Embracing or resisting discourses of learning and being a learner}

While we have a theoretical interest in connecting learning and work discourses and practices, we are also mindful of the tensions in conflating them. In an earlier research project ('Uncovering Learning at Work') these tensions came to the fore. In that project we examined ways in which learning occurs in and through everyday work in different sections of a large educational provider with the aim of finding ways that this learning could be harnessed more effectively. As outlined in Boud and Solomon (2003), we found a number of political and identity tensions amongst the research participants in terms of understanding and naming themselves as learners as well as workers. Some employees openly resisted the identity, for example:

I take on board wherever I can whatever I can, without thinking about it as learning... I don't see myself as being a learner. I am happy with what I know at the moment and whatever comes along I'll use if it's appropriate.

In the study, the workers' designated position, and their recognition and power within their working group or the organisation were important factors in the uptake (or not) of learning and learner discourses. This finding drew our attention to the ways the terms 'learning' and 'learner' have been used in research on workplace and organisational learning as if they were unproblematic in relation to workers' identities, and as if there was shared meaning about what they referred to. 
In our current research there has been a different uptake of work and learning discourses illustrated in the following quotes by electrical workers at SupplyCo:

Teaching someone something is actually really important in reinforcing your own things and learning.

So to put it mildly I try and encourage a learning environment. I myself and perhaps my history ..., and I've worked with a number of mentors who have actually encouraged me through my entire career about learning. Sure what we did today, tomorrow we could better because we know more tomorrow. Again, I emphasis the key word there is 'learning'.

These comments demonstrate that in this organisation learning and being a learner appear to be accepted. We found no resistance and learning was not associated immediately with formal education and training processes. This led us to speculate about what was occurring at SupplyCo that was different from the previous organisation in which the language of learning and being a learner was circumscribed. What could we understand from the practices of SupplyCo that illuminated the ways in which learning was being deployed? What made it okay to identify as learning and being a learner in SupplyCo?

\section{The research site and research methods}

SupplyCo is one of four organisations in which we have been identifying and describing a range of IDPs (Chappell et al, 2009) to contribute to understandings of the learning work of organisational practices. SupplyCo (a pseudonym) is a stateowned public utility organisation in NSW, Australia, which has been moving towards developing a more corporatised (eg self-insured) identity over recent years. Together with providing power they offer a range of related services to over 800,000 customers, they maintain a grid across 24,000 square kilometres and they respond to emergencies.

The regional SupplyCo group we worked with was further organised into functional areas, two of which were: transmission and distribution. Transmission is concerned with taking high voltage power and reducing its voltage to meet needs of households and industry. Distribution, as the name suggests, is concerned with distributing the reduced voltage power. While we spoke to some regional managers, for the most part our data comes from workers within the Distribution function of SupplyCo.

Data collection occurred over six months in 2008. We conducted 28 semi-structured interviews and participated in many less formal interactions with other workers during SupplyCo events (including Safety Days, barbeques, in lunch rooms and other social spaces). We also facilitated focus groups with SupplyCo employees, and we reviewed organisational documents (including organisational charts, job descriptions, work activity forms, etc). Following thematic and broad discourse analysis of the data we met with SupplyCo managers to present and discuss findings in terms of potential implications and usefulness for the organisation in the future.

\section{Conceptual framework}


The principal conceptual emphasis of our study is on practice theory. This has been adopted as it provides a means of acknowledging that work involves complex bundles of doings and sayings that bring together the individual, the social and the organisational to produce effects. It enables us to see organisations as involving groups of practices that can be understood as such by the actors involved, but it also provides ways of theorising what is occurring over time and space.

For our feedback sessions with SupplyCo managers to discuss findings we felt that conceptualisations regarding practice that we have been using were not directly relevant to the organisation as we had entered the site emphasising a focus on learning. We sought a way of framing learning that would on the one hand be familiar to them, while on the other hand would introduce what we felt were new ideas and ways of understanding learning in their organisation. We chose Delors' (1996) idea of the four pillars of education, taken from an educational, lifelong learning perspective. This enabled us to discuss learning, but also relate it to work practices. The framework was well-received by the management group and offered a lens for understanding and, as we were told in a follow-up conversation, promoting the wider learning benefits of a range of IDPs within the organisation.

\section{Practice theory and organisational practices}

There is an increasing interest in exploring and theorising practice in contemporary social theory. The 'practice turn' has been named to highlight this shift in theorising about social phenomena, including organisations (see for example Schatzki (2005; 2006;); Gherardi (2000); Gherardi et al. (1998); Nicolini et al. 2003, Kemmis 2005). It brings to the fore conceptions that all human activity including 'knowledge, meaning, science, power, language and social institutions' are part of and constitute the 'field of practices' (Schatzki et al., 2001). The practice turn seeks to steer clear of theoretical dualities (for example, individual/social; structure/agency, etc), rather grounding thinking and theorising in practices as the 'primary building block of social life and meaning' (Schatzki et al., 2001). Schatzki sees practices as 'culturally constituted, meaningful action' (2001:2), and he suggests that practices are 'embodied, materially mediated arrays of human activity centrally organised around shared practical understanding'.

The usefulness of a practice approach to this research in particular, and to organisational studies in general, is that it interconnects the individual and the social (for our purposes, the workplace) and meaning with the material. Organisations, rather than being relatively stable entities, are always dynamically unfolding and changing - 'organisations as they happen' (Schatzki 2006). They are 'bundles of practices and material arrangements' (Schatzki, 2006) or 'systems of practices' (Gherardi, 2000), and this practice approach positions the worker and the social context of work, and the organisation, as mutually produced where knowing and doing cannot be separated (Gherardi, 2000). Exploring our data using these underpinning ideas, we have been able to draw out this mutual production as it occurs in our research sites. This has enabled us to uncover ways in which organisational practices become shared, enmeshed, carried forward and at the same time changed.

Schatzki (2006) understands practices as consisting of elements of both structure and action. Structure includes understandings of the 'how to' of a practice, the rules, 
possible ends and goals as well as more general understandings. Action is about the carrying out of a practice in specific time-space contexts. The already existing practice structures that encompass organisations frame action possibilities. Practices are understood to be carried forward within the practice memory of an organisation (its organisational memory) together with workers enacting them (Schatzki, 2005; 2006; Schatzki et al., 2001). In enacting organisational practices, workers' understandings of those practices (structure-action elements) become enmeshed with previous understandings of similar practices from other contexts - in this way practices are perpetuated and at the same time varied (Schatzki, 2006). For Schatzki (2006), learning is viewed as a crucial element in the perpetuation and change of organisational practices, but learning is discussed only briefly and relates closely to transfer of knowledge or know how. While the work of Schatzki and colleagues does not particularly focus on varieties of learning, the view of organisations as dynamic, where organisational practices persist and simultaneously change, can be re-presented to draw out different kinds of learning, and organisational practices as sites of individual and organisational learning can be usefully interrogated.

From practice theory, and the work of Schatzki in particular, we take up the idea that workers co-construct organisational practices and create shared meaning and understandings of those practices. At SupplyCo the IDP, safety practices, had been going through significant change; one key area of change was from what had been primarily a practice involving regular audits through standardised checklists carried out by managers 'on' workers, to a practice of jointly negotiated discussions between managers and workers - sometimes initiated by workers themselves. The interviewees' comments reconstructed the 'old' practice in relation to the new safety observations thereby highlighting the ongoing structure elements, new actions and the mutual production of workers, the social context of work and the organisation itself.

Put a different way, workers are learning the how-to, the contextual characteristics and interrelationships of practices. At the same time, organisational practices play a role in framing what is learned, how it is learned and by whom. In the following section, we outline the learning (in organisational practices) framework we employed to characterise learning IDPs in SupplyCo.

\section{Four pillars of learning: knowing, doing, being and living together}

In 1996 Delors submitted a report from the International Commission on Education for the Twenty-first Century Learning: The Treasure Within to the United Nations Educational, Scientific and Cultural Organization (UNESCO). The concepts central to Delors' report were four interrelated 'pillars of education': learning to know; learning to do; learning to be; and, learning to live together (Delors 1996:37).

The teasing out and naming of different kinds of learning is always going to be problematic and open to criticism. However, Delors' work opens up ways of thinking about learning that may be new to some including, we suggest, many who understand work as made up of knowing and doing. Further, the pillars' framework is 'easy to use and to apply flexibility in any context' (de Leo 2006:3). Indeed, for some, the appeal of mobilising the framework is because it is simple. 
The pillars cut across and extend traditional education and training (and learning) categories and understandings. Although the framework was developed to promote lifelong learning, for us it provides a helpful (and simple) heuristic for discussing workplace learning, one that enables a focus on a shift beyond the more traditional knowledge and skills focus associated with work. It is the inter-relatedness of the pillars that has been useful for us in our exploration of learning in relation to IDPs at SupplyCo.

The first two pillars are very familiar in workplaces (learning to know and learning to do) whereas the latter two (learning to be and learning to live together) are usually discussed in relation to our lives more holistically. In a general sense, the idea of learning to know is about knowledge acquisition. It also refers to, '... learning to learn, so as to benefit from opportunities education provides throughout life' (Delors 1996:37). Learning to do helps people 'acquire not only an occupational skill but also, more broadly, the competence to deal with many situations and work in teams' (Delors 1996:37). Learning to be is purported to 'develop one's personality and be able to act with even greater autonomy, judgement and personal responsibility' (Delors 1996:37). It recognises that learning takes place in a social context and that the concept of learners is culturally loaded, and notions of learning and being are not universally understood. More recently, education and learning have been acknowledged as sites of self-work and the concept of identity has become central to much of this discussion. Learning to live together is the fourth pillar with a focus on developing 'an understanding of other people and an appreciation of interdependence - carrying out joint projects and learning to manage conflicts - in a spirit of respect for the values of pluralism, mutual understanding and peace' (Delors 1996:37).

In our research we have adapted Delors' pillars to relate specifically to the workplace context. At SupplyCo workers were learning to know about the industry, about the organisation, about electricity supply, about safety, and about a range of other 'things'. The second pillar, learning to do was evidenced in a variety of ways too: learning to do particular tasks such as how to climb a pole; how to behave in meetings, what to do at the depot, etc. For the most part, there was nothing especially new in this regard. We suspected that managers and workers already knew that everyone gained skills and knowledge as part of their everyday work. However, it was the third and fourth pillars (learning to be and learning to live together), and their inter-relatedness with the former two, that provided new ways of thinking about organisational practices. These pillars drew attention to how workers were learning to become a lineworker, a project officer, an operations manager, responsible and accountable employees, a safe SupplyCo worker and more. Further, we were also able to draw attention to how SupplyCo workers were learning to be together by appreciating the diversity in others' perspectives, and of others' roles within the organisation, communicating with a wide range of colleagues, and so on.

In our exploration of interviewees' descriptions of the IDP, safety practices, we have been able to draw out and comment on the learning that we suggest is going on involving one or a combination of the pillars. This is perhaps important in workplaces such as SupplyCo where the focus has traditionally been on skills and knowledge acquisition. Thus, for example, we were able to describe how safety practices not only increased workers' skills and knowledge, but also how people were learning to be 
different kinds of SupplyCo workers as well as learning to work together in and across work teams/areas.

\section{Safety practices at SupplyCo}

The main IDPs we found at SupplyCo were performance appraisal, projects, acting up and safety. While all these practices were explored, safety practices caught our attention and became a key focus in this case study. Safety notices were posted on almost every surface of the organisation, demonstrating that safety was integral to the work, and to the workers. Handling electricity is hazardous and appropriate measures need to be taken, however the attention to safety at SupplyCo permeated beyond legislative and policy compliance. Attention to safety was embedded in cultural, operational and detailed understandings of safety and safety practices throughout the organisation.

For example, safety is experienced and deployed by SupplyCo through mandated work activities, employee forums/discussions, processes, performance indicators, and through the completion of forms and other written procedures. First, through mandated work practices, many employees are required to perform hazard and risk assessments, and safety audits and observations. Second, SupplyCo holds forums for training and discussion and they have instituted special safety days. Third, processes, such as the activities and conversations involved in hazard and risk assessments, and safety audits and observations, also acted as a means of embedding safety within the organisation. Fourth, performance indicators such as lost time frequency rate that specifies targets compound safe organisational performance. Finally, an array of documents records the results of hazard and risk assessments, and safety audits and observations, which can be used to further improve awareness, compliance and performance. Attention to safety is not only an aspiration of management, it is also heard in formal interviews with SupplyCo employees, in our informal discussions, during meetings, as well as during social gatherings. Indeed, safety matters to the people in this organisation as much as it matters to the organisation itself - as one employee told us:

It's a sign of the times ... I don't think they want you to get hurt ... and it's in your face all the time.

A distinctive component of safety practices at SupplyCo are safety observations which occur on almost a daily basis. The ways in which safety observations are carried out, who is involved, and how they are approached and viewed by SupplyCo workers, have undergone significant changes in recent times. In the following section we focus on safety observations, examining how interviewees talked about them.

\section{Safety observations}

One worker describes safety observations as:

[We] go out and observe our teams and just go through what they're doing. The leading hand inducts us on to the site and tells us who the site co-ordinator is and if there are any isolations. We go through the permits and whatever you've 
got to sign on, the hazard risk assessments, they take us all through that. Then we just observe how they're working.

This way of enacting safety observations is relatively new and constitutes a notable departure from the already existing safety audits. Where safety audits had operated for many years as a compliance measure to 'catch out' unsafe behaviour of workers and unsafe equipment, safety observations foreground social relationships and emphasise teamwork and shared commitment. In the interview extract above the team leader's comments demonstrate informality, valuing of the workers' contributions and a lack of, or at least understated hierarchical roles. Even the re-naming from 'audits' to 'observations' suggests changes in purposes and relationships. The following comments draw out some of the changes to safety practices, particularly the shift from a context of disciplining (us and them) to one of mutual involvement and respect (us together):

In previous days we've done audits and we write up the audit and tell them what they've done wrong, and it's all the negative stuff.

In an audit, you can have a non-conformance and tell somebody they have to fix something up and whatever. You can do a similar thing in an observation, but generally what you try and do is first go in and praise a person for all the right things they're doing, and suggest to them "Oh do you think that might have been a better way to do it"? and see their comment, and also ask them if they've got any ways that they think, you know, safety could have been improved.

The two workers quoted above connect the past safety practice of audits with the present safety practice of observations. The second comment, in particular, demonstrates how the new observations still include some aspects of the past audits. In other words, following Schatzki, there is a recounting of ' $a$ ' possible performance of safety observations where understanding and persistence of previous practices become enmeshed, shared and carried forward with new understandings producing changes to safety practices. The doings and sayings (embedding knowledge) are becoming part of the organisational practice memory comprising what safety practices mean at SupplyCo.

Another employee also alludes to past audits in his description of observations as different in terms of being 'softer', and he emphasises the communicative and social relationships - 'a good way of... meeting the teams and ...talking to them.' At the same time he stresses the importance of safety practices in terms of collaborative commitment to achieve an important organisational effect:

It's a softer observational way. And, safety observations are a good way of also getting out and meeting the teams and you know, talking to them, and raising the awareness of safety, because of safety statistics, so, there's a reason for it, there's a driver for it, to remove, you know, to reduce the number of accidents, and that's, everything we all have in our heart, we don't want to have to go back and report to you know, someone's family that they've injured themselves or they've died.

Whereas safety audits were strictly hierarchical in terms of who was doing the auditing and who was the audited, safety observations at SupplyCo cut across 
organisational hierarchies. The organisation is working towards having all employees carry out safety observations. The ways interviewees discursively constructed safety observations as collegial practices implicates an ongoing shift - Schatzki's action possibilities - towards this goal.

Rather than feeling threatened by safety observations, some SupplyCo workers welcomed them. The reason may be that they enabled discussions between workers from across the organisation. This, in turn, enabled workers to pick up information about aspects of SupplyCo - as one worker says:

I believe if you talk about an issue, and ... if something else isn't in the back of your mind, you'll bring it forward and have a chat about it, so it is making opportunities there ... if a designer's out there doing a safety observation and the guy doesn't like something about design, he'll certainly tell him then, whereas the guy, one would never have been out there [if] we weren't doing safety observations and the two wouldn't have a conversation if he did go out there with them. But now he's having those conversations and he's picking up information.

Picking up new information is part of a broader benefit of building relationships and finding opportunities to communicate beyond the immediate, task-oriented work. The new safety practice was sometimes even initiated by workers themselves:

What I've found really good about it is the guys respond to it, the guys in the field. If you were doing an audit on them, they'd be like "Oh god, not another audit" but you walk up and they go "Are you doing a safety ob today?" and you'll be going "I wasn't going to" and they go "Well can I tell you something anyway?" and they'll actually tell you things.

These extracts recounting people's experiences of safety observations at SupplyCo demonstrate that this practice goes a long way beyond the organisational purposes of ensuring safety compliance. The statements describing how safety observations are enacted foreground their evolution from audits that had particular structure/action elements to ones where some of the structures were maintained, such as the overall ends and goals (fewer injuries and deaths) and some of the rules (eg concerning legislative compliance). It is these existing practice structures that persist and frame the present and possible future safety practices. The extracts also situate safety observations as actions, that is, the carrying out of the practice in specific time-space contexts. In relation to safety observations, the extracts presented above demonstrate how workers understand the changes working and look towards future changes as well.

If safety observations are practices that both persist and change already existing practices, then what can we say about the learning that might be going on and how and why is it okay to be learning and a learner? One partial answer could be that if organisations are always 'happening', and organisational practices are complex bundles of doings and sayings that change (Schatzki 2006), then it should be okay to identify as learning. However, we want to be able to say more than this, and below 
we further analyse safety observations in terms of the inter-relationship of Delors' four pillars of learning.

Safety practices at SupplyCo are being enacted in ways that involve knowledge and skills while at the same time they foreground learning to be and learning to be together as important components of organisational practices. In each of the interview extracts above there is evidence of employees being new kinds of workers and working together in new ways - new communication and collaboration practices are being tried and learned. We heard that as part of the safety observations workers are asked if they've got any ways that they think, you know, safety could have been improved; that observations are a good way of also getting out and meeting the teams and you know, talking to them, and if something else isn't in the back of your mind, you'll bring it forward and have a chat about it, so, it is making opportunities there. Indeed, the bottom up nature of these learning to be and learning to be together practices is demonstrated by you walk up and they go "Are you doing a safety obs today"? and you'll be going "I wasn't going to" and they go "Well can I tell you something anyway?" and they'll actually tell you things.

The workers are not simply positioned as auditors and audited enacting safety observations that are concerned with performance or non-performance, they are learning new skills and knowledge and, importantly they are learning to relate in ways that construct different identities - ones that offer ideas and information as experts in their field, and ones that are more collegial and where communicating as a team becomes as much as what it means to be a SupplyCo worker as being a skilled linesperson.

Notably, even though 'learning' and 'learner' are not named in the interview extracts cited above, notions of learning and being a learner sit comfortably with SupplyCo employees. There were more explicit discourses of learning and being a learner in interviewee comments. For example, both workers and managers found safety observations useful as learning reminders as well as opportunities to make change happen:

Yeah it always just reinforces - like you just take things for granted but those things just refresh your memory and make you think about them for a while ... It just makes you think. Like you do it anyway but you just think about it.

The learning culture is explicitly referred to and can be summed up in this final extract:

Yeah, yeah, yeah. We go through an investigation process and work out the whys and what nots and what happened and how it happened and how we can improve things to make it not happen again. You know everyone's learning and nobody wants to deliberately make mistakes. I think that's the way you've got to manage it. Yeah you can be ...you stupid bastard what did you do that for sort of thing.

\section{Discussion and conclusion}

Safety practices, and safety observations in particular, unambiguously fulfilled the criteria for being understood as an IDP. Further, the unembarrassed and sometimes explicit way in which learning was considered at SupplyCo was a refreshing contrast to what we had observed in our earlier study. Safety observations had not only been 
adopted, they had been embraced by all we encountered at SupplyCo. Learning was embedded as an accepted part of a necessary function of the organisation, and the discourse of workers reflected this.

This leads to the question of why is the discourse of learning taken up in one place but not another? Why was it not seen in an organisation in which learning was central to its mission, but was observed in an organisation whose mission was to deliver electricity? At the simplest level, this occurred because learning, at least learning safety practices, was accepted as a legitimate part of everyday work at SupplyCo. It was integrated and valued in everyday work practices. It was not regarded as alien, as something that occurs in formal settings or as requiring teachers or trainers or only articulated when managers were present. Workers accepted that they were in the business of learning safety - it had become part of the practice memory of the organisaton. While the need to protect themselves provided a strong personal incentive, it is not always the case that safety practices can be readily accepted in very risky industries as Somerville and Lloyd's (2006) studies of the mining industry dramatically testify.

In the previous study organisation where the acceptance of a learning discourse at work was more problematic, learning was seen primarily as something that employees' clients (students) were assisted to do, not as something that applied to themselves. Learning and learners were terms used very frequently, but in the context of what others did. Being a learner was not accepted as a legitimate position for a worker (as a teacher) as it made one vulnerable and not a full part of the workforce. It may be that proximity to learning as something serviced by the organisation inhibited take up of the discourse by workers. Enhancing students' learning was the key purpose of the organisation, thus learning was embedded in the enactment of organisational practices. However, there was not an IDP that embedded employees' learning; it was not part of the practice memory of the organisation.

The four pillars of learning were embraced by management in SupplyCo as they resonated with their view of the organisation they were creating. These aspects of learning could be seen and integrated as part of their practice and the discourse of work. They recognised, when introduced to the idea, that learning to be and learning to live together were part of their enterprise. They understood before we arrived that they were promoting learning as an integrated part of work, and our language and explication enabled them to articulate this more fully.

Of course, this study does not address the question of how an organisation is enabled to take up learning in this way. However, it does show that learning can be inserted successfully into organisational practices in ways that mean that it is okay to learn and be a learner at work.

\section{References}

Bereiter, C. (1994). "Constructivism, socioculturalism and Popper's world". Educational Researcher, Vol. 23, No. 7, pp. 21-3.

Boud, D. (2006). "Combining work and learning: the disturbing challenge of practice”. In R. Edwards, J. Gallacher and S. Whittaker. (Eds.). Learning Outside 
the Academy: International research perspectives on lifelong learning. London: Routledge, pp. 77-89.

Boud, D. and Solomon, N. (2003). "'I don't think I am a learner': Acts of naming learners at work", Journal of Workplace Learning, Vol. 15, No. 7-8, pp. 326-331.

Chappell, C., Scheeres, H., Boud, D. and Rooney, D. (2009). "Working out work: integrated development practices in organizations". In J. Field, J. Gallacher, and R. Ingram (Eds.) Researching Transitions in Lifelong Learning, London: Routledge, pp. $175-188$

de Leo, J. (2006). Social and cultural perspectives of ESD: Cultural diversity and intercultural understanding within ESD. UNESCO Expert Meeting on Education for Sustainable Development (ESD): Reorienting education to address sustainability, NESCO APNIEVE Australia, Thailand, 1-3 May, 2006.

Delors, J. (Chair) (1996). Learning: The treasure within. Report to UNESCO of the International Commission on Education for the Twenty-First Century. Paris: UNESCO Publishing.

Ellström, P. E. (2001). "Integrating learning and work: Problems and prospects", Human Resource Development Quarterly, Vol. 12, No. 4, pp. 421-435.

Engeström, Y. (2001). "Expansive learning at work: toward an activity theoretical reconceptualization", Journal of Education and Work, Vol. 14, No. 1, pp. 133-156.

Fuller, A. and Unwin, L. (2002). "Developing pedagogies for the contemporary workplace", in K. Evans, P. Hodkinson and L. Unwin (Eds) Working to Learn; Transforming learning in the workplace, London, Routledge, pp. 95-111.

Gherardi, S. (2000). "Practice-based theorizing on learning and knowing in organizations". Organization, Vol. 7, No. 2, pp. 211-223.

Gherardi, S., Nicolini, D., and Odella, F. (1998). "Towards a social understanding of how people learn in organizations: The notion of situated curriculum". Management Learning, Vol. 29, No. 3, pp. 273-297.

Guile, D. and Griffiths, T. (2001). "Learning through work experience", Journal of Education and Work, Vol. 14, No. 1, pp. 113-131.

Hodkinson, P. (2004). "Research as a form of work: Expertise, community and methodological objectivity”, British Educational Research Journal, Vol. 30, No. 1, pp. 10-26.

Kemmis, S. (2005). "Knowing practice: Searching for saliences". Pedagogy, Culture and Society, Vol. 13, No. 3, pp. 391-426.

Lave, J and Wenger, E (1991). Situated Learning; Legitimate peripheral participation, Cambridge, Cambridge University Press.

Nicolini, D., Gherardi, S., and Yanow, D. (2003). "Introduction: Toward a practicebased view of knowing and learning in organizations". In D. Nicolini, S. Gherardi and D. Yanow (Eds.), Knowing in Organizations: A practice-based approach M.E. Sharpe, Armonk, NY, pp. 3-31.

Poell, R, Chivers, G. and Van der Krogt (2000). "Learning network theory: Organising the dynamic relationships between earning and work", Management Learning Vol. 31, No. 1, pp. 25-49.

Saugstad, T. (2005). "Aristotle's contribution to scholastic and non-scholastic learning theories", Pedagogy, Culture and Society, Vol. 13, No. 3, pp. 347-66.

Schatzki, T.R. (2006). "On organizations as they happen". Organization Studies, Vol. 27, No. 12, pp. 1863-1873.

Schatzki, T.R. (2005). "Peripheral vision: The sites of organizations". Organization Studies, Vol. 26, No. 3, pp. 465-484. 
Schatzki, T.R., Knorr Cetina, K., and von Savigny, E. (Eds.) (2001). The Practice Turn in Contemporary Theory. Routledge, London and New York.

Senge, P. M. (1990) .The Fifth Discipline: The art and practice of the learning organization, New York: Doubleday.

Skule S and Reichborn A (2002). Learning-Conducive Work: A survey of learning conditions in Norwegian workplaces, CEDEFOP, Luxembourg.

Somerville, M. and Lloyd, A. (2006). "Codified knowledge and embodied learning: the problem of safety training”, Studies in Continuing Education, Vol. 28, No. 3, pp. $279-28$. 